\title{
O CAMPO DA ATENÇÃO PSICOSSOCIAL: FORMAR E CUIDAR NO CURSO DE QUALIFICAÇÃO NA ATENÇÃO DIÁRIA EM SAÚDE MENTAL (CBAD)
}

THE FIELD OF PSYCHOSOCIAL CARE:TRAINING AND CARING IN THE TRAINING COURSE ON DAY-CARE IN MENTAL HEALTH

Pilar Belmonte ${ }^{1}$

Resumo Este texto se propõe a contribuir para a discussão acerca da formação de profissionais de saúde segundo os objetivos da Reforma Psiquiátrica. Para tanto, apresenta o caminho percorrido no Brasil desde o surgimento dos primeiros movimentos da Reforma no País, a construção do novo modelo assistencial vigente, alternativo ao modelo hospitalocêntrico, assim como a experiência do Curso de Qualificação na Atenção Diária em Saúde Mental (CBAD), que objetiva a formação de cuidadores em saúde mental. Discute também possíveis estratégias e propostas que garantam uma formação articulada com a prática, refletindo-se no cuidado multidisciplinar diferenciado e no alcance da maior autonomia possível da clientela atendida.

Palavras-chave reforma psiquiátrica; cuidadores em saúde mental; educação permanente em saúde.
Abstract This article furthers the discussion on the training of health professionals according to the guidelines of the Psychiatric Reform by reviewing the steps taken by the country from the appearance of the first reform movements until the development of the current care model, an alternative to hospital-centered models. The article also presents the experience acquired in the Training Course on Day-Care in Mental Health, whose objective is to train health care professionals in mental health. Moreover, it also discusses possible strategies and proposals for assuring that the received training also involves practice, which would be reflected in differentiated multidisciplinary care and in a possibly larger autonomy of clients.

Key words psychiatric reform; mental health care providers; permanent education in health. 


\section{Breve histórico da assistência psiquiátrica}

Chegou-se ao século XXI com grandes conquistas no campo da saúde mental. Hoje são notoriamente reconhecidos os serviços diários como os centros de atenção psicossocial (CAPS), as oficinas terapêuticas e os residenciais terapêuticos. Entretanto, junto a este já não tão novo modelo, que veio se consolidando nos últimos vinte e cinco anos, ainda se convive com o modelo hospitalar, que persiste em nosso país refletindo o modelo clássico psiquiátrico nascido na França em fins do século XVIII.

No dia-a-dia dos asilos psiquiátricos, que até hoje perduram em função deste mesmo modelo, a vida dos internos vem sofrendo mudanças desde a criação do primeiro hospital psiquiátrico brasileiro, o Hospício D. Pedro II, na Praia Vermelha, em 1852. São raras, hoje, as imagens, comuns no final da década de 1970, de loucos vagando nus por pavilhões de hospícios.

O passo para a mudança tem origem no Movimento de Trabalhadores em Saúde Mental (MTSM), que dá início ao processo da Reforma Psiquiátrica. Desde o final da década de 1970, quando foi constituído o MTSM, várias experiências e iniciativas foram realizadas visando a superar a tradição manicomial.

Em meados da década de 1980, tais propostas de reformulação psiquiátrica foram se firmando, ocupando um lugar sólido no quadro das políticas de saúde. Como marcos deste processo de mudanças registram-se as Conferências Nacionais de Saúde Mental (CNSM), a primeira em 1987, a segunda em 1992 e a última em 2003, nas quais a saúde e a doença mental passam a ser discutida não apenas por profissionais, mas também por usuários e familiares, envolvendo a todos como agentes no processo. Outro momento histórico é o II Encontro Nacional dos Trabalhadores de Saúde Mental em Bauru, em 1987, nascido a partir da I CNSM e responsável pela reorganização do movimento, que se tornaria um movimento social mais global - e não mais apenas restrito aos trabalhadores em saúde mental- e assumiria o lema "Por uma sociedade sem manicômios".

A intervenção na Casa de Saúde Anchieta, em Santos, em 3 de maio de 1989, possibilitada pelo contexto das políticas no campo da Reforma Sanitária, abre um novo campo de estratégias no âmbito das políticas de saúde mental. Um primeiro manicômio é progressivamente desmontado para, em seu lugar, nascerem instituições, serviços e práticas verdadeiramente substitutivas ao modelo psiquiátrico asilar.

Entrementes, surge, em 1989, o projeto de lei Paulo Delgado, o qual propõe a substituição progressiva dos manicômios por outras práticas terapêuticas e discute a cidadania do louco, dando continuidade ao debate não apenas entre os profissionais de saúde, mas em toda a sociedade. Esse projeto, que sofreu diversos substitutivos, foi finalmente aprovado em 2001 e, se não conseguiu manter em seu texto a definitiva substituição dos ma- 
nicômios, constituiu-se, mesmo assim, em um marco. A lei $\mathrm{n}^{\circ} 10.216$, de 06 de abril de 2001, é a primeira que “dispõe sobre a proteção e os direitos das pessoas portadoras de transtornos mentais e redireciona o modelo assistencial em saúde mental" a ser aprovada, prevendo que o tratamento deve ser oferecido, preferencialmente, em serviços comunitários de saúde mental, da forma menos invasiva possível.

A discussão estende-se e, em 1990, é assinada a Declaração de Caracas, na qual se pode destacar a orientação de se diversificar a oferta de serviços, visando a desmistificar a crença de que o hospital psiquiátrico seria o local ideal para o tratamento das pessoas com transtornos mentais e também a reforçar que esse hospital, enquanto continuar existindo, deve excluir, de suas práticas, o confinamento e o martírio oferecendo tratamento digno e humano.

No Brasil, além dos cinco núcleos de atenção psicossocial (NAPS), surgidos como alternativas substitutivas à Casa de Saúde Anchieta, no início dos anos 1990 - comprovando na prática a possibilidade de superação do modelo manicomial -, outras propostas começaram a ser construídas.

Em São Paulo, já havia sido inaugurado, em 1987, o centro de atenção psicossocial batizado de CAPS Prof. Luís da Rocha Cerqueira, que funciona até hoje e é considerado o primeiro serviço de atenção diária em saúde mental no Brasil2.

Outras iniciativas, como os centros de convivência, as cooperativas, os lares abrigados (ou serviços residenciais), buscam facilitar o desenvolvimento de aptidões sociais dos usuários e, conseqüentemente, de sua autonomia.

Essas experiências comprovam que o manicômio é prescindível como local de tratamento, deixando claro que a loucura é polissêmica, cada indivíduo é diferente dos demais - essas novas formas de atendimento permitem que se trabalhe com as diferenças.

O país conta hoje com 723 CAPS, sendo 63 no Rio de Janeiro, e 294 serviços residenciais terapêuticos (SRT), 49 no Rio de Janeiro, que abrigam egressos dos hospitais psiquiátricos. No país, há 1.624 moradores nos serviços residenciais, sendo 238 no Estado do Rio de Janeiro (Saúde Mental no SUS, 2005). Esses egressos podem receber uma bolsa incentivo oferecida pelo governo federal, que foi instituída pela lei $\mathrm{n}^{\mathrm{o}} 10.708$, de 2003, e estabeleceu o auxílio reabilitação psicossocial para pacientes acometidos de transtornos mentais egressos de internação psiquiátrica. O município do Rio de Janeiro oferece também uma bolsa incentivo, que não pode ser cumulativa com a bolsa oferecida pelo governo federal e que também provê o pagamento de valor mensal ao egresso do hospital psiquiátrico ou ao seu responsável.

Deve-se também apontar a extinção progressiva dos leitos psiquiátricos e o descredenciamento de clínicas, de 'fábrica de loucos', pelo governo federal, através da Coordenação Geral de Saúde Mental do Ministério da Saúde. 
O país, que contava, em 1991, com 85.037 leitos em hospitais psiquiátricos próprios e conveniados, contava em 2005 com 43.092 leitos (Saúde Mental no SUS, 2005).

No âmbito desses projetos já concretos, surge, em 1995, como proposta complementar, o Programa de Apoio a Desospitalização (PAD), o qual previa a alta administrativa de pacientes com longos períodos de hospitalização, reencaminhando-os às suas famílias de origem, com o repasse dos recursos federais para divisão entre as famílias e as secretarias municipais de saúde.

Naquele momento, não foi possível implementar o PAD. Hoje, dez anos depois, como já mencionado, contamos com programas em nível nacional e municipal que, através do repasse de recursos financeiros às famílias e/ou ao próprio paciente, permitem que ele abandone sua 'hospedagem' em um hospital psiquiátrico e possa retornar à sua família ou morar por conta própria ou nos residenciais terapêuticos.

Apesar de, na época, a proposta do PAD não ter encontrado recursos técnicos que a tornassem factível, ela foi a força motora para se pensar em uma proposta de capacitação de acompanhantes terapêuticos que pudessem dar suporte não apenas ao paciente, egresso de longa internação, mas também às famílias que os estariam recebendo de volta e que teriam dúvidas sobre como proceder.

Surge, assim, o Curso Básico de Acompanhamento Domiciliar (CBAD), que em 2005, já com a nova denominação - Curso de Qualificação na Atenção Diária em Saúde Mental -, completou uma década formando cuidadores em saúde mental.

\section{O CBAD}

Considerando esta proposta de cuidados de atenção diária em saúde mental, surge o projeto do acompanhante domiciliar (AD), que propõe a formação de acompanhantes domiciliares, atuais cuidadores. Pensa-se nesta pessoa de referência como alguém que pode vir a contribuir na viabilização da possibilidade de suporte social, desempenhando um papel de ligação sujeito-família, sujeito-serviço, sujeito-comunidade. Ao cliente não hospitalizado ou desospitalizado deve ser oferecida uma série de possibilidades assistenciais, como tem ocorrido nos núcleos e centros de atenção psicossocial, hospitais dia/noite, residenciais terapêuticos, oficinas e pensões protegidas, unidades psiquiátricas em hospitais gerais, cooperativas de trabalho, centros de conveniência.

O complexo trabalho de desinstitucionalização requer, na maioria das vezes, um esforço, uma dedicação e uma organização assistencial muito maior, mais elaborada e dinâmica do que a psiquiatria costuma oferecer. É nesse 
sentido que se inclui o cuidador, tornando-se uma pessoa-chave nesta proposta. Sua tarefa fundamental é estar ao lado de determinados clientes que se encontram em estado de maior dificuldade ou em momentos de crise, tornando-se a referência para o suporte a ser oferecido a essas pessoas e às suas famílias, acompanhando-os ora nas tarefas do dia-a-dia e no deslocamento casa-serviço/serviço-casa, ora em atividades de lazer, estudo e trabalho.

O cuidador deve funcionar dentro do universo do paciente. Não tem limitação espacial; funciona onde requer o cliente, aliviando ansiedades, garantindo um tratamento mais humano, personalizado, ajudando a estruturar hábitos, visando sempre à autonomia do cliente.

Assim, o CBAD visa a propiciar a qualificação, através de atividades teóricas e práticas, de cuidadores em saúde mental para agirem como facilitadores nas relações entre cliente-família, cliente-serviço, cliente-comunidade, atuando não só junto a clientes que retomam suas próprias vidas após anos de segregação em hospícios - e que, portanto, exigem um acompanhamento na reintegração ao novo ambiente -, mas também junto a novos casos de mal-estar psíquico, garantindo o cuidado de atenção diária nos moldes propostos pela Reforma Psiquiátrica.

O curso em questão, oferecido pela Escola Politécnica de Saúde Joaquim Venâncio, Fundação Oswaldo Cruz (EPSJV/Fiocruz), não só integra como complementa as mudanças que vêm ocorrendo na assistência psiquiátrica em nosso país. Cabe ressaltar que é uma experiência inovadora, sendo pioneira na capacitação de técnicos para o projeto de desinstitucionalização em curso no país, dividindo-se em dois momentos: um teórico e outro prático.

A parte teórica consta de aulas expositivas, através de palestras, vídeos, seminários, dinâmicas, ministradas por professores da Fiocruz e convidados, visando a transmitir um grau de informação que possibilite ao aluno 'situar-se' no campo da saúde mental, entender sua evolução, compreendendo a finalidade de sua participação e percebendo, em conjunto com a coordenação, sua adequação para atuar como cuidador.

Ao final da parte teórica, apenas os alunos considerados aptos passam à parte prática, que é composta por estágios em serviços de cuidados diários em saúde mental, como os CAPS.

A supervisão é uma atividade fundamental para o trabalho do cuidador. O principal objetivo da supervisão é auxiliar a resolver as dificuldades internas do cuidador, relacionadas à tarefa e à troca constante de informações. A supervisão fica a cargo do coordenador do serviço no qual o cuidador estiver realizando o estágio, e da coordenação do curso, que promove encontros quinzenais durante a fase do estágio para acompanhamento das atividades.

O curso tem a duração total de cinco meses, tendo a parte teórica duração aproximada dois meses, com carga horária de 140 horas, e a parte prática, cerca de três meses, totalizando 260 horas. 
Os temas abordados nas aulas discutem desde a questão histórica da construção das práticas em saúde mental, passando pela organização dos serviços de saúde mental, até as relações humanas, abordando a equipe de trabalho e a família. Apresentam-se também os principais quadros clínicos e terapêuticas, incluindo-se a psicofarmacologia e as práticas grupais, que são as mais utilizadas nos CAPS. Ocorrem ainda, durante o curso, visitas a alguns serviços de atenção diária, para que, antes do estágio, o aluno já possa conhecer e ter noção do tipo de serviço, da clientela e das atividades que fazem parte do dia-a-dia dos CAPS.

O principal material didático utilizado é o livro Temas em saúde mental, (EPSJV, 2003) elaborado pela equipe do Grupo de Trabalho em Saúde Mental da EPSJV/Fiocruz com o objetivo de servir de suporte bibliográfico para os cursos de saúde mental, principalmente para o CBAD.

Exige-se apenas o primeiro grau completo para que os interessados possam se candidatar a uma das 25 vagas oferecidas anualmente. A seleção consta de análise de currículo e entrevista, na qual se expõem os objetivos do curso e tenta-se verificar o interesse e a adequação do candidato às atribuições previstas.

Apesar de não se exigir formação específica na área da saúde, verificase nas dez edições do curso uma grande procura por parte de profissionais de saúde de nível médio, como os técnicos de enfermagem. A seguir, apresenta-se um breve resumo dos egressos do CBAD.

\section{Perfil dos egressos}

Incluindo-se a turma de 2005, que concluiu recentemente o CBAD, formaram-se desde o primeiro curso 198 alunos. Desse total, 18,3\% são homens e $81,7 \%$, mulheres, refletindo a realidade dos serviços de saúde, em que a maioria dos trabalhadores de nível médio é do gênero feminino, conseqüência histórica da formação da área de enfermagem, que, em seu início, pensava-se como uma profissão a ser exercida exclusivamente por mulheres, já que exigia condições entendidas como intrínsecas ao gênero.

Apesar de a exigência mínima ser o ensino fundamental, a escolaridade dos alunos concluintes concentra-se no ensino médio (65\%). Com apenas o ensino fundamental, temos $9 \%$, e com nível superior, $26 \%$.

Quanto à inserção no mercado de trabalho e na profissão, temos 66,3\% dos alunos do curso inseridos no mercado de trabalho, muitos deles na área de saúde, enquanto que apenas 33,7\% não trabalhavam.

A formação concentra-se em mais de 50\% na área da enfermagem, sendo $29,6 \%$ auxiliares de enfermagem e $25,3 \%$ técnicos de enfermagem. Os demais $(45,1 \%)$ dividem-se entre as mais variadas profissões, incluindo-se algumas 
da área da saúde, como enfermagem, terapia ocupacional e serviço social.

Há uma grande demanda por parte não apenas dos CAPS, mas também de serviços de saúde vinculados a empresas e de familiares por cuidadores formados no CBAD. Assim, a coordenação tem disponibilizado uma relação dos concluintes para os serviços públicos e privados de saúde mental e para as famílias que a procuram demandando cuidadores.

O município de Niterói ilustra a importância do CBAD, visto que, na proposta de criação de um quadro de cuidadores para atuarem nos serviços locais de saúde mental, incluiu como exigência, na seleção, a conclusão deste curso. Dessa forma, atualmente, há diversos egressos exercendo as atribuições de cuidadores nos CAPS e residenciais terapêuticos do estado do Rio de Janeiro, o que não apenas serve como estímulo para a continuidade do trabalho, mas também aponta a necessidade de se ampliar e expandir essa experiência, já fortemente consolidada.

\section{Propostas e desafios}

A conjuntura atual não poderia estar mais propícia para se discutir os vieses entre formação e assistência. Está claro que tanto os serviços substitutivos ao hospital psiquiátrico quanto à formação de cuidadores em saúde mental estão seguramente concretizadas. Além disso, na área de educação e saúde, a portaria $\mathrm{n}^{\circ} 198$ do Ministério da Saúde (MS), de 13 de fevereiro de 2004, que "institui a Política Nacional de Educação Permanente em Saúde como estratégia do Sistema Único de Saúde para a formação e o desenvolvimento de trabalhadores para o setor" (MS, 2004a), prevê a integração entre as ações e o ensino da saúde, evitando uma verticalização da tomada de decisões, para promover a educação permanente em saúde, agregando o aprendizado, a reflexão crítica sobre o trabalho, a resolutividade da clínica e a promoção da saúde.

Para o alcance desses objetivos, pode-se usar a estratégia dos pólos de educação em saúde, que se originam dos já existentes pólos de educação em saúde da família.

Os pólos são pensados como instâncias de articulação entre os serviços, os gestores, as instituições formadoras e as instâncias de controle social. Não são locais de execução, mas, sim, espaços privilegiados para identificar necessidades, articulando o diálogo entre os diferentes atores para superar a formação tradicional. São espaços de negociação que estão se constituindo nos estados, regiões e municípios. Podem fazer parte desses pólos os gestores de saúde e de educação, as instituições de ensino (universidades, escolas técnicas e de saúde pública e centros formadores) que tenham cursos na área de saúde, núcleos de saúde coletiva, hospitais de ensino, serviços de saúde, estudantes e trabalhadores da área de saúde, conselhos de saúde e 
movimentos sociais que tenham entre seus temas questões referentes às políticas públicas de saúde.

No campo da saúde mental, também há portarias que enfatizam a importância da formação de recursos humanos, como a de $\mathrm{n}^{\circ} 816$, de 30 de abril de 2002, que prevê um programa de capacitação de recursos humanos para os CAPS-ad - (CAPS álcool e outras drogas), incluindo a locação de recursos financeiros, e a anterior, de $\mathrm{n}^{\circ} 336$, de 19 de fevereiro de 2002, que trata, entre outros assuntos, da capacitação para os CAPS.

Em uma circular de 08 de janeiro de 2004, as coordenações de Saúde Mental e de Gestão da Atenção Básica discutem a inclusão das ações de saúde mental na atenção básica. Nesse documento, a formação aparece como uma estratégia prioritária para que se concretize a inclusão da saúde mental na atenção básica através dos pólos de educação permanente em saúde.

O documento sugere cursos voltados para as equipes da rede básica, tanto de nível superior como de nível médio, de acordo com os princípios da Reforma Psiquiátrica, tendo-se em mente que essa capacitação deve auxiliar na promoção da maior autonomia possível dos usuários. Na circular, é apresentado também o modelo do apoio matricial de saúde mental às equipes de atenção básica.

É notório que a proposta do Programa de Saúde da Família (PSF) foi um avanço que propiciou maior resolutividade dos problemas da população por meio da vinculação com equipes. Destas, 56\% alegaram já ter realizado alguma ação em saúde mental (MS, 2004b). Entretanto, nem sempre elas se sentem em condições de responder a essa demanda, seja pela ausência de recursos humanos, seja pelas próprias características da formação das equipes, que carecem de uma capacitação específica na área de saúde mental.

Considerando-se semelhanças entre as propostas dos CAPS e da atenção básica, em termos do papel estratégico que ambos desempenham nas respectivas áreas, da organização por território e da noção de rede, não é de se estranhar que as equipes dos CAPS (ou de outros equipamentos de saúde mental, na ausência destes) sejam identificadas como o apoio matricial às diferentes equipes de atenção básica, provendo-as de suporte técnico na área de saúde mental, compartilhando casos, em co-responsabilização, excluindo a lógica do encaminhamento, já que se espera, com essa parceria, desenvolver ações conjuntas e ampliar a capacidade resolutiva pelas equipes locais.

Apresentado de forma breve esse contexto, pode-se partir para uma reflexão acerca da formação do trabalhador de saúde mental.

Propõe-se uma aprendizagem que articule trabalho e ensino, com o envolvimento de todos os atores mencionados. O objetivo é uma educação permanente, através da aprendizagem significativa, que ultrapasse a clássica organização disciplinar por atividades, a qual estabelece o que o aluno deve 
aprender e reproduzir, e que ofereça conteúdos com relevância para a prática profissional que possam transformar a realidade social através da problematização do processo de trabalho.

Necessita-se, então, problematizar esta prática. Se, em um primeiro momento, o CAPS surge para se contrapor ao hospital psiquiátrico como uma alternativa e, mais adiante, como uma substituição, espera-se mais dele no momento. Não há dúvidas da extrema importância desse espaço como um local de acolhimento, de resgate da individualidade e da cidadania. Entretanto, sua missão não deve ser pensada apenas como a de um lugar que trata, que hospeda, que provê desde os cuidados básicos como alimentação e asseio, até a medicação e o lazer. O CAPS deve ser indutor de práticas e organizador de uma rede que inclua os serviços de saúde mental dos hospitais gerais, os ambulatórios, as residências terapêuticas, o Programa de Saúde da Família, as cooperativas, enfim, a própria sociedade.

O CAPS, hoje, não é a única opção. Ele faz parte de uma rede de atenção psicossocial, e os papéis de todos os diferentes dispositivos de assistência em saúde mental devem ser pensados conjuntamente.

Mais do que isso, já que a palavra do momento é integralidade, devese pensar a saúde em articulação com outros elementos importantes para a qualidade de vida e o controle social.

Há que se discutir também, na avaliação desses serviços, a criação de instrumentos que possam, em algum nível, mensurar o impacto e a resolutividade das ações neles executadas.

Para se alcançar uma educação permanente e adequada aos trabalhadores do campo da atenção psicossocial, deve-se pensar no perfil desse trabalhador, nas competências que ele deve ter, para não só acolher, mas ajudar o usuário, em qualquer dispositivo em que esteja ele, a alcançar a autonomia possível.

Há que se analisar os problemas, as dificuldades, as ameaças com que o profissional se depara em seu processo de trabalho. A transformação da prática e da organização do trabalho ocorre através da problematização do próprio processo de trabalho.

Todos os projetos, todas as propostas, tendem, cada vez mais, a respeitar a diversidade das regiões, os processos de gestão de serviços, ou seja, as diferenças.

É necessário estabelecer uma articulação com as instituições formadoras de todos os tipos com interesse e cursos na área de saúde mental, com os profissionais dos serviços de atenção psicossocial, assim como os gestores municipais de saúde mental e demais envolvidos, para oferecer cobertura e apoio aos municípios, desenvolvendo iniciativas de integração de conteúdos, cursos modulares, ofertas flexíveis de capacitação de acordo com as necessidades locais e das próprias equipes no que concerne à formação em saúde mental. 
Poder-se-ia, ainda, organizar grupos de trabalho que pudessem, em um primeiro momento, fazer o levantamento da força de trabalho nos serviços, para posteriormente ampliar a discussão, envolvendo a mais variada gama de atores, sobre as atividades exercidas nos serviços, sobre as novas propostas que busquem a integralidade da atenção e o alcance da autonomia, a problematização do processo de trabalho, para alcançar propostas que superem as concepções hegemônicas da formação.

Sabe-se que a formação de trabalhadores para a área de saúde mental ainda não se realiza hegemonicamente, voltada para os serviços de atenção diária.

Inicia-se, ainda de forma tímida, a discussão de novos modelos curriculares para a graduação. Os profissionais de nível médio e elementar não têm uma capacitação específica para a área e, durante a sua formação, poucos são apresentados às novas formas de atenção em saúde mental, ainda cumprindo estágio em psiquiatria em instituições de características asilares. No entanto, segundo a portaria $\mathrm{n}^{\circ} 336$ do MS, de 2002, os CAPS devem ter, no mínimo, quatro profissionais de nível médio para um atendimento de 20 usuários por turno. Levando-se em conta o número de CAPS já existentes até dezembro de 2005, temos um contingente de mais de 2.500 trabalhadores em nível nacional que não tiveram em sua formação um treinamento específico para atuar nesse tipo de serviço.

O desafio, baseado na experiência de sucesso do CBAD, seria descentralizar o curso, respeitando-se as características regionais e as discussões dos pólos de educação permanente, para, em conjunto, discutirem-se propostas que garantam a qualificação desses trabalhadores já envolvidos no dia-a-dia para a execução de atividades que possam ter um impacto maior na consolidação da autonomia e na ressocialização do egresso de internações psiquiátricas. Isto só será possível se o trabalhador puder contar com ferramentas que o instrumentalizem nas suas tarefas diárias.

\section{Considerações finais}

Novos serviços trazem novas soluções e também novos problemas. A capacitação de recursos humanos, a avaliação e a inserção desses serviços na rede de cuidados à saúde são algumas das questões que se colocam neste novo contexto.

Há necessidade de se fomentar uma ampla discussão, com a participação de todos os profissionais vinculados ao SUS e às instituições de ensino e pesquisa, para elaboração de instrumentos que possam responder ao desafio de avaliar as estratégias de tratamento oferecidas pelos CAPS e outras formas de atendimento extra-hospitalar. 
Para se implantar uma nova forma de atendimento em saúde, são necessários, obviamente, profissionais capacitados a trabalhar nesta nova lógica de atenção. O profissional de saúde deve estar preparado para fazer frente a uma variedade de situações e de possibilidades de atuação que devem se articular visando à solução dos problemas de saúde.

Identificar esse contingente de trabalhadores que se encontra exercendo suas atividades na assistência à saúde mental e discutir as possibilidades que, através da formação, possam contribuir para oferecer uma atenção integral e resolutiva aos portadores de sofrimento psíquico, com objetivo do alcance da autonomia, é um desafio.

A formação de recursos humanos para os CAPS, serviços residenciais terapêuticos e outros serviços de base comunitária - que hoje acontece nos próprios serviços, no momento da prática e de maneira informal - deve ser pensada de maneira mais ampla e permanente para possibilitar a criação de uma cultura onde o respeito e o reconhecimento da diferença estejam presentes em todos os profissionais de saúde, já que, de uma forma ou de outra, todos lidam com a clientela dentro de uma proposta de atenção multidisciplinar, a qual prevê uma equipe integrada nas atividades e compartilhando conhecimento.

Espera-se que a discussão aqui iniciada e a experiência acumulada em dez anos na formação de cuidadores possam ser compartilhadas para o alcance da maior autonomia possível do sujeito portador de transtornos mentais - objetivo maior dos profissionais de saúde mental, encontrem-se eles na educação ou na assistência.

\section{Notas}

${ }^{1}$ Professora da Escola Politécnica de Saúde Joaquim Venâncio, da Fundação Oswaldo Cruz. Mestre em Saúde Coletiva pelo Instituto de Medicina Social da Universidade do Estado do Rio de Janeiro (UERJ) < pilarb@fiocruz.br>

2 Os primeiros NAPS de Santos funcionavam em regime de 24 horas, todos os dias da semana, e faziam parte de uma rede de estruturas externas substitutivas ao asilo. Os CAPS se organizaram desde seu início em regime diurno, funcionando em média oito horas por dia, de segunda a sexta-feira. Entretanto, essa distinção não se manteve, e é comum encontrarmos serviços denominados NAPS que funcionam no mesmo regime dos CAPS, tendo em comum a busca de um modo de assistência caracterizado por um serviço de atenção diária, que prescinda da internação, e a organização em unidades regionalizadas, que trabalham com o modelo de reabilitação psicossocial, com oficinas e atividades em grupo, desvinculadas do espaço hospitalar. A portaria $\mathrm{n}^{\circ} 336 / 02$, que define os CAPS por diferentes níveis de complexidade, gerou uma tendência à unificação da nomenclatura utilizada. 


\section{Referências}

BARROS, Denise D. 1994. Jardins de Abel: desconstrução do Manicômio de Trieste. São Paulo: EdUsp.

BELMONTE, Pilar; LAGRANGE, Valéria. 1995. Projeto do Curso Básico de Atenção Diária em Saúde Mental (CBAD). (Mimeo).

COSTA, Jurandir F. 1989. História da psiquiatria no Brasil: um corte ideológico. $4^{a}$ ed. Rio de Janeiro: Xenon.

EGGERS, José Carlos. 1985. O acompanhante terapêutico: um recurso técnico em psicoterapia de pacientes críticos. Revista de Psiquiatria do Rio Grande do Sul, v. 7, n. 1, (45-58).

EPSJV (Escola Politécnica de Saúde Joaquim Venâncio) (org.). 2003. Textos de apoio em saúde mental. Rio de Janeiro: Editora Fiocruz. (Série Trabalho e Formação em Saúde).

.2005. Políticas de saúde Rio de Janeiro: Editora Fiocruz. (Série Trabalho e Formação em Saúde).

GOLDBERG, Jairo. 1994. A clínica da psicose: um projeto na rede pública. Rio de Janeiro: Te Cora.

MS (Ministério da Saúde). 2003a. Seminário Diretrizes sobre Política de Saúde - Saúde Mental. Brasília. (mimeo) .2003b. Seminário Diretrizes sobre Política de Saúde - Álcool e Drogas. Brasília. (mimeo) .2004a. Portaria $\mathrm{n}^{\circ} 198$, de 13 de feve- reiro de 2004. Institui a Política Nacional de Educação Permanente em Saúde como estratégia do Sistema Único de Saúde para a formação e o desenvolvimento de trabalhadores para o setor e dá outras providências.

.2004b. Saúde Mental e Atenção Básica. O Vínculo e o Diálogo Necessários. Inclusão das Ações de Saúde Mental na Atenção Básica. Circular Conjunta $\mathrm{n}^{\circ}$ 01, de 08/01/04. Ministério da Saúde/ Secretaria de Atenção à Saúde: Brasília. .2004c. Saúde mental no SUS: os Centros de Atenção Psicossocial. Brasília. .2004d. Legislação em Saúde Mental. $5^{\text {a }}$ ed. Brasília: Ministério da Saúde.

NICÁCIO, Maria Fernanda de S. 1994. O processo de transformação da saúde mental em Santos: desconstrução de saberes, instituições e cultura. Dissertação de Mestrado, São Paulo:Faculdade de Psicologia, Pontifícia Universidade Católica de São Paulo.

ROTELLI, Franco et al. 1990. Desinstitucionalização. São Paulo: HUCITEC.

SAÚDE MENTAL NO SUS. Informativo em Saúde Mental. 2005. v. 4, n. 21. Disponível em: <www.inverso.org. br/blob/160.pdf $>$. Acesso em: 22 mar. 2006.

SAVIO, Domingos. 1995. Loucuras que se realizam. Jornal do Brasil, 8 de jan., Revista de Domingo.

Recebido em 19/12/2005

Aprovado em 06/03/2006 